\title{
Mechanical Deformations in Smectic-C Main-Chain Liquid-Crystalline Elastomers
}

\author{
Antoni Sánchez-Ferrer ${ }^{1,2}$ and Heino Finkelmann ${ }^{2}$ \\ ${ }^{1}$ Physics Department, University of Fribourg, Fribourg, Switzerland \\ ${ }^{2}$ Institute for Macromolecular Chemistry, Albert Ludwigs University, \\ Stefan-Meier-Str., Freiburg, Germany
}

\begin{abstract}
A novel crosslinked smectic-C Main-Chain Liquid-Crystalline Elastomer (MCLCE) has been synthesized by polycondensation of vinyloxy-terminated mesogens, tetramethyldisiloxane and pentamethylpentaoxapentasilecane. The introduction of the functional vinyloxy group allows the synthesis of well-defined networks having low soluble content and good mechanical properties. Networks having a macroscopic uniformly ordered director and a conical distribution of the smectic layer normal with respect to the director are mechanically deformed by uniaxial and shear deformations. Under uniaxial deformations two processes were observed: parallel to the director the mechanical field directly couples to the smectic tilt angle while perpendicular to the director a reorientation process takes place. A shear deformation parallel and perpendicular to the director causes $a$ uniform layer orientation and the network exhibits a smectic- $C$ monodomain phase having a macroscopic uniform director and layer orientation. This process is reversible for shear deformation perpendicular and irreversible by applying the shear force parallel to the director.
\end{abstract}

Keywords: liquid-crystalline elastomers; shear deformations; uniaxial deformations; $\mathrm{X}$-ray diffractometry

\section{INTRODUCTION}

Smectic elastomers, due to their layered structure, exhibit distinct anisotropic mechanical properties and mechanical deformation processes parallel or perpendicular to the smectic layer normal causing different responses of the networks. Besides the anisotropy of the mechanical moduli, reorientation processes are of interest. The most

Address correspondence to Antoni Sánchez-Ferrer, Physics Department, University of Fribourg, Chemin du Musée, 3, 1700 Fribourg, Switzerland. E-mail: antonio. sanchez-ferrer@unifr.ch 
important aspect is whether an external mechanical field parallel and perpendicular to the smectic layer normal couples either to the orientational order of the mesogenic units, the director, or to the layer orientation. Up to date only Side-Chain Liquid-Crystalline Elastomers (SCLCEs) with smectic-A (SmA) and smectic-C (SmC) phases have been analyzed with respect to their anisotropic mechanical properties.

In the case of macroscopically ordered SmA SCLCEs, uniaxial mechanical deformations have been performed parallel and perpendicular to the director [1-3]. While the first deformation process causes layer reorientation above a characteristic threshold stress, the second process leaves the structure unmodified and indicates the in-plane fluidity of the smectic layers [4-5]. SmC elastomers, significant because of their optical and ferroelectric properties such as in the case of chiral molecules ( $\mathrm{SmC}^{*}$ phase) [6], have also been synthesized. By applying a multi-stage crosslinking process, macroscopic uniformly ordered networks are now realizable [7-10].

So far experiments on the deformation of SmC SCLCEs do not indicate any changes of the tilt angle between the director and the layer orientation. The basic question is, whether Main-Chain LiquidCrystalline Elastomers (MCLCEs), where the mesogenic units are directly incorporated into the polymer backbone, differ in their mechanical response.

In this work, the mechanical response of new SmC MCLCEs will be described under uniaxial or shear deformations which are applied parallel or perpendicular to the director. The orientation and reorientation processes of the $\mathrm{SmC}$ elastomer are studied to identify whether the director and/or layers couple with the applied external mechanical field. The $\mathrm{SmC}$ elastomer is a monodomain with respect to the director orientation but has a conical orientation distribution of the layer normal with respect to the director as deduced from the X-ray patterns.

\section{RESULTS AND DISCUSSION}

\subsection{Synthesis of MCLCEs}

The smectic-C main-chain liquid-crystalline elastomer (SmC-MCE2.5) was prepared using the spin-casting technique, by mixing $2.5 \mathrm{~mol} \%$ of the isotropic crosslinker, the chain extender, $5 \mathrm{~mol}-\%$ of the azobenzene dye and $95 \mathrm{~mol}-\%$ of the mesogen, all together in toluene. After the non-complete hydrosilylation reaction, the sample was removed from the reactor and aligned by applying a uniaxial stress. For the completion of the hydrosilylation reaction, the network 

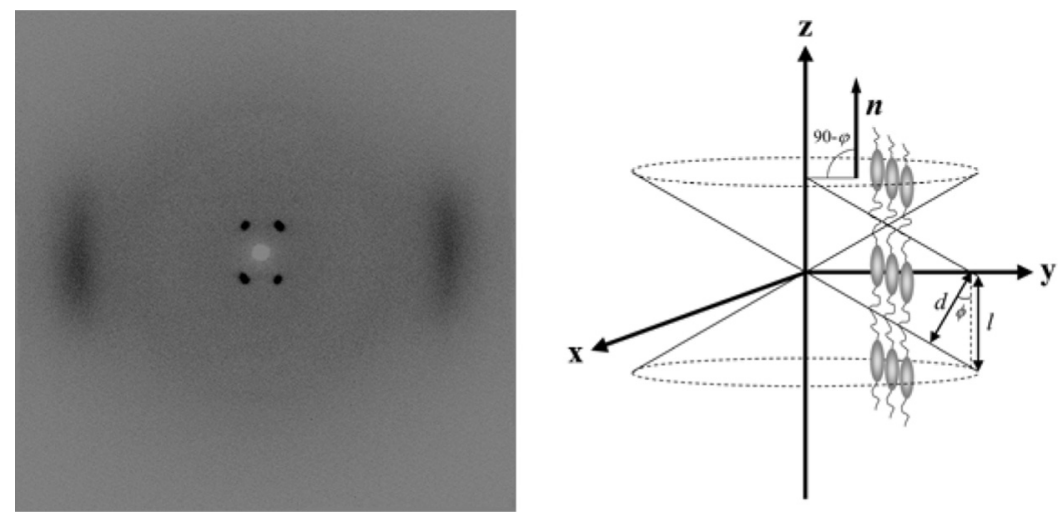

FIGURE 1 X-ray picture of the SmC-MCE-2.5 sample at $25^{\circ} \mathrm{C}$ in the $\mathrm{SmC}$ phase showing a conical layer distribution structure of a $\mathrm{SmC}$ main-chain elastomer. In this picture the descriptions of the distances $(d)$ and angles $(\phi, \varphi)$ of monomers and layers and length $(l)$ between repeating units are shown.

was cured under load during the second step of the crosslinking reaction in order to align and fix the liquid crystal phase.

With respect to the director alignment X-ray measurements (Figure 1) reveal a well-oriented sample having an order parameter of $S=0.78$ at $25^{\circ} \mathrm{C}$. The sample exhibits a conical layer distribution structure as schematically presented in Figure 1. The mesogens are oriented in the $z$-direction and the layers are distributed conically about $z$. This structure is elucidated from the X-ray experiment and yields the mesogen distance of $4.5 \AA$, layer distance $d=25 \AA$ and layer angle $\phi=30^{\circ}$. The calculated length of the monomer unit is $l=29 \AA$, which is close to the all-trans length of $34 \AA$ (Fig. 1).

\subsection{Uniaxial Deformations}

The main question is whether MCLCEs differ compared to SCLCEs on applying an external mechanical field parallel or perpendicular to the director, and whether this force couples either to the director and/or to the layer orientation. In Figure 2, uniaxial stress-strain experiments are depicted, where deformations were performed parallel and perpendicular with respect to the director on the sample having the conical layer distribution structure.

Under deformation parallel to the director, two different linear regions can be observed. In the first region, for strain values of 1.00 $<\lambda<1.20$, the sample has a Young's modulus of $E_{\mathrm{z}}=13 \mathrm{MPa}$. In the second region, with strain values of $\lambda>1.20$, the network shows 


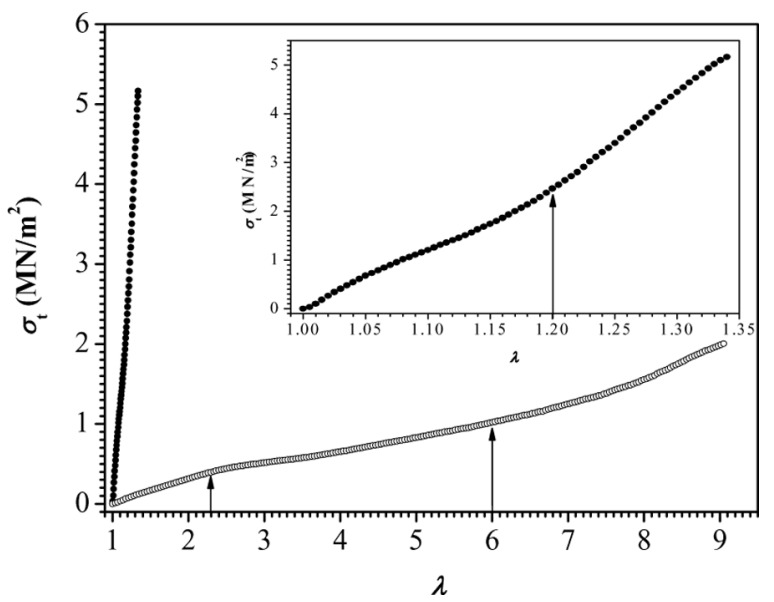

FIGURE 2 Uniaxial stress-strain curves parallel (O) and perpendicular $(\bigcirc)$ to the director on a conical layer distribution structure of the elastomer $\mathrm{SmC}-\mathrm{MCE}-2.5$ at $25^{\circ} \mathrm{C}$ in the $\mathrm{SmC}$ phase. (The arrows indicate regions of changing slope; refer to text). Inset: uniaxial stress-strain curve parallel to the director.

a stiffer behaviour with a Young's modulus of $E_{\mathrm{z}}=20 \mathrm{MPa}$. This deformation is a reversible process, where the sample completely recovers its initial state on unloading.

In the deformation perpendicular to the director three regions can be differentiated, the first region with strain values of $1.00<\lambda<2.30$, the second at $2.30<\lambda<6.00$ and the third at $\lambda>6.00$. The slopes of these three regions are $\mathrm{d} \sigma / \mathrm{d} \lambda=0.3,0.2$ and $0.4 \mathrm{MPa}$, respectively. This deformation along the $y$-axis is a non-reversible process. The initial state can only be recovered by stretching the sample in the $z$-axis.

Uniaxial strain parallel to the director revealed two different moduli above and below a deformation of about 30\%, which might indicate different deformation mechanisms. The non-stretched sample exhibits four reflections in the small-angle region indicating a tilt angle of the director with respect to the layer normal of $30^{\circ}$ (Fig. 3). The two maxima of the azimuthal intensity distribution in the wide-angle region reveal a uniform director orientation and an order parameter of $S=0.82$. For the elastomer strained by $20 \%$, the most relevant change in the structure concerns the tilt angle $\phi$, which increased from $31^{\circ}$ to $40^{\circ}$, while the orientation of the director remains constant and parallel to the applied stress. Furthermore, the intensity distribution of the wide-angle reflections narrows indicating an increase of the order parameter from 0.82 to 0.89 . 

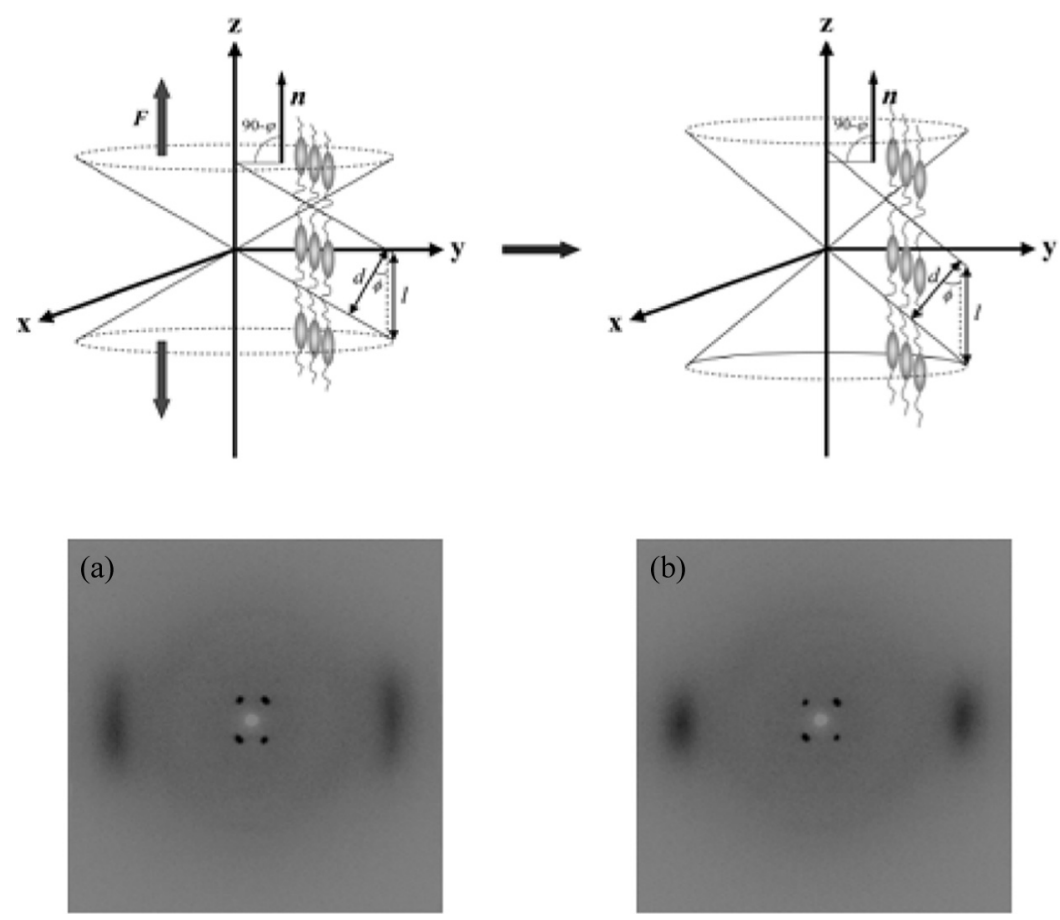

FIGURE 3 Uniaxial deformation process parallel to the director on a $\mathrm{SmC}$ MCLCE and X-ray pictures at (a) $\lambda=1.00$ and (b) $\lambda=1.20$.

For this system, however, the change of the tilt angle, which is equivalent to a layer rotation, might have the origin in an optimization of the packing of the mesogenic monomer units under strain. It has to be noted that a coupling between mechanical field and tilt angle has not been identified by experiments for side-chain elastomers so far (Fig. 3).

In the uniaxial stress perpendicular to the director, the mechanical measurement of the network already indicates a completely different response with a low slope value for the uniaxial strain perpendicular to the director and resembles the stress-strain behavior of SmA side-chain elastomers. For these SmA elastomers, the deformation perpendicular to the layer normal does not affect either the layer orientation nor the director orientation and reflects the in-plain fluidity of the smectic phase structure [2].

Starting from the non-stretched conical layer distribution structure with four reflections in the small-angle region and two in the wide-angle region, actually a continuous layer rotation is observed towards an uniform orientation of the layer normal in the plane 
perpendicular to the applied stress at $\lambda=2.30$. Obviously the mechanical field couples to the smectic layers and removes defects of the initial conical layer distribution structure. Consistent with this layer arrangement is the observation of four reflections in the wide-angle regime, which characterizes the tilt of the $\mathrm{SmC}$ phase.

In the strain regime of $2.30<\lambda<6.00$, where the stress-strain curve is almost linear (Fig. 2), a slight layer rotation of the layer normal occurs. Most interestingly the director simultaneously rotates by about $90^{\circ}$ and indicates that the mechanical field causes an increase of the tilt angle. Above $\lambda>6.00$, in the third regime, the director and layers become redistributed towards a conical layer distribution structure with a tilt angle of about $40^{\circ}$. In this strain regime, the mechanical field couples to the director and produces a structure as observed for the deformation process parallel to the director. This reorientation process (Fig. 4) is also reflected in the order parameter, which recovers the initial value of $S=0.85$, as observed for the deformation process parallel to the director.
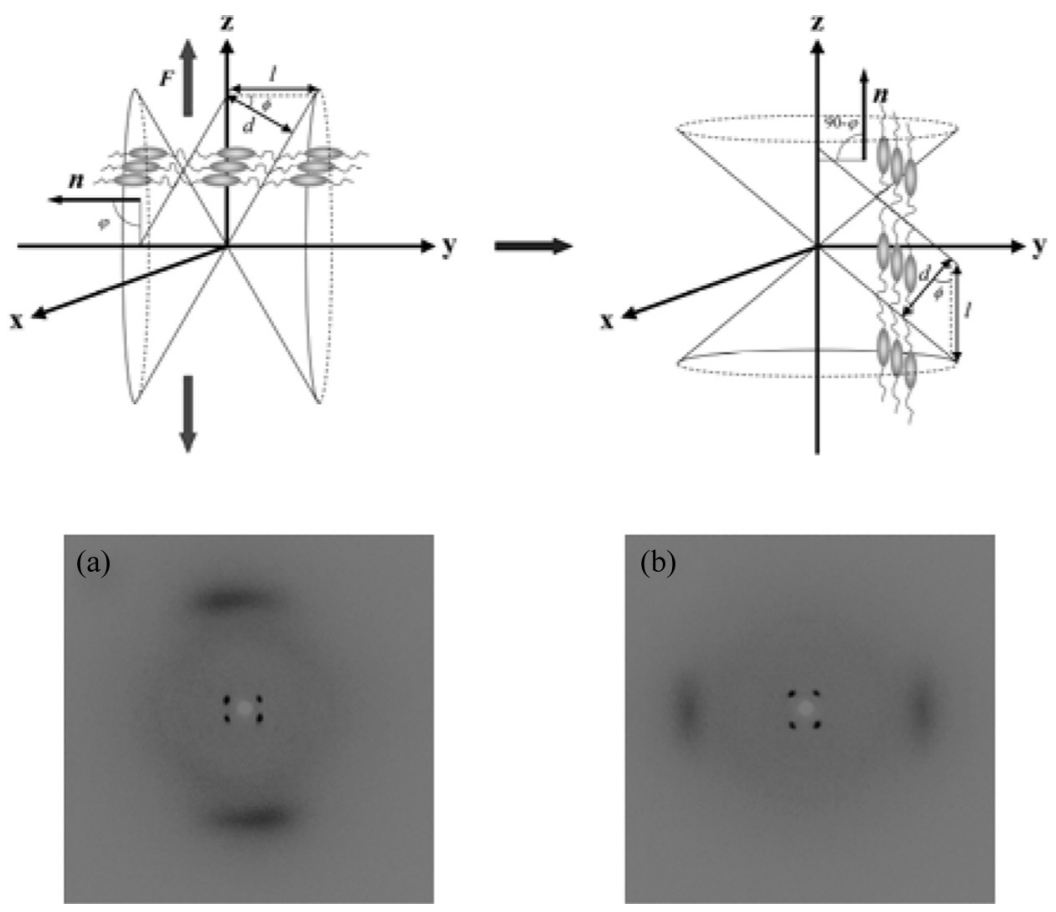

FIGURE 4 Uniaxial deformation process perpendicular to the director on a SmC MCLCE and X-ray pictures at (a) $\lambda=1.0$ and (b) $\lambda=8.0$. 


\subsection{Shear Deformations}

Experiments on SmC side-chain elastomers with conical layer distribution structure already demonstrated that a shear field transforms a conical layer distribution structure into a monodomain possessing a uniform layer and director orientation [8-9]. The basic question is, whether the SmC main-chain elastomers exhibit a similar behaviour.

In Figure 5, where the shear stress $\tau$ is plotted versus the shear angle $\gamma$, the results are presented for the shear deformation parallel and perpendicular to the director of the network with conical layer distribution structure. Interestingly for both deformation modes, at the beginning of the shear deformation $\left(\gamma<21^{\circ} ; 0.37 \mathrm{rad}\right)$ the same shear modulus of $G \sim 0.3 \mathrm{MPa}$ was found. This might indicate that both shear geometries cause the same reorientation process. For the shear deformation perpendicular to the initial director orientation above this angle, a local soft response occurs in the regime between $19^{\circ}(0.34 \mathrm{rad})<\gamma<21^{\circ}(0.37 \mathrm{rad})$. Above this angle again a linear response of the shear stress $\tau$ occurs with about the same modulus. For the shear deformation parallel to the initial director orientation above $\gamma>24^{\circ}(0.42 \mathrm{rad})$ the film wrinkles upon further shear deformation and the deformation process becomes irreversible. The elastomer does not recover its original shape on unloading.

Starting from the initial sample with the conical layer distribution structure, the shear stress perpendicular to the director couples to the

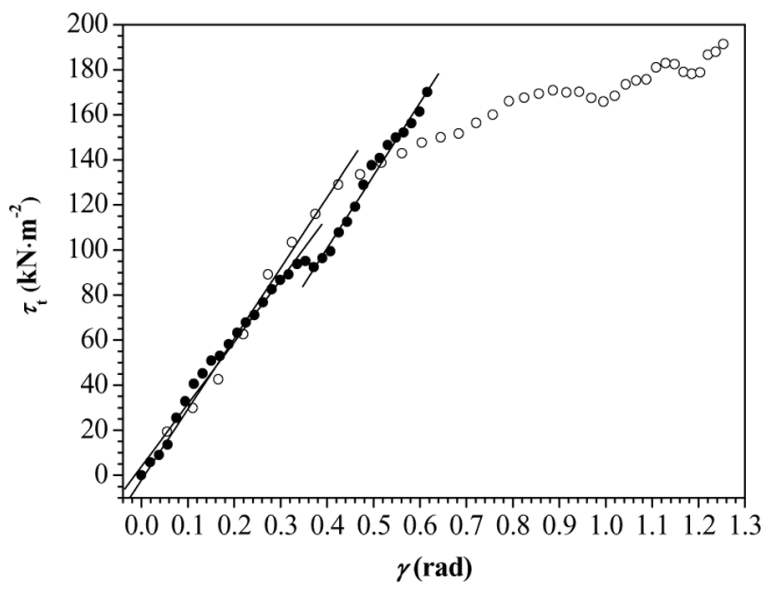

FIGURE 5 Shear stress-strain curves perpendicular $(\bigcirc)$ and parallel $(\bigcirc)$ to the director on a conical layer distribution structure of the elastomer $\mathrm{SmC}-\mathrm{MCE}-2.5$ at $25^{\circ} \mathrm{C}$ in the $\mathrm{SmC}$ phase. 
director as well as to the layer orientation and causes a continuous rotation of the director and the layers with the shear angle. This process, however, preferentially affects those layers, where the smectic layer normal can rotate towards the shear force. At about $\gamma \sim 25^{\circ}$, which corresponds to the dip in the shear stress-strain curve (Fig. 5), all layers are completely removed that are not aligned in the shear direction.

Additionally it has to be noted that this reorientation process directly affects the intrinsic tilt angle, which increases continuously from $37^{\circ}$ to $41^{\circ}$ and remains unaffected at larger shear strain. It resembles the uniaxial strain behaviour of the elastomer parallel to the director, which also increases the tilt up to a constant angle (Fig. 6).

Applying the shear deformation parallel to the director also causes a reorientation leading to a monodomain structure. This deformation process, however, basically differs from the previous experiment. The shear strain does not affect the director orientation, which remains fixed parallel to the shear direction (Fig. 7). Only those layers
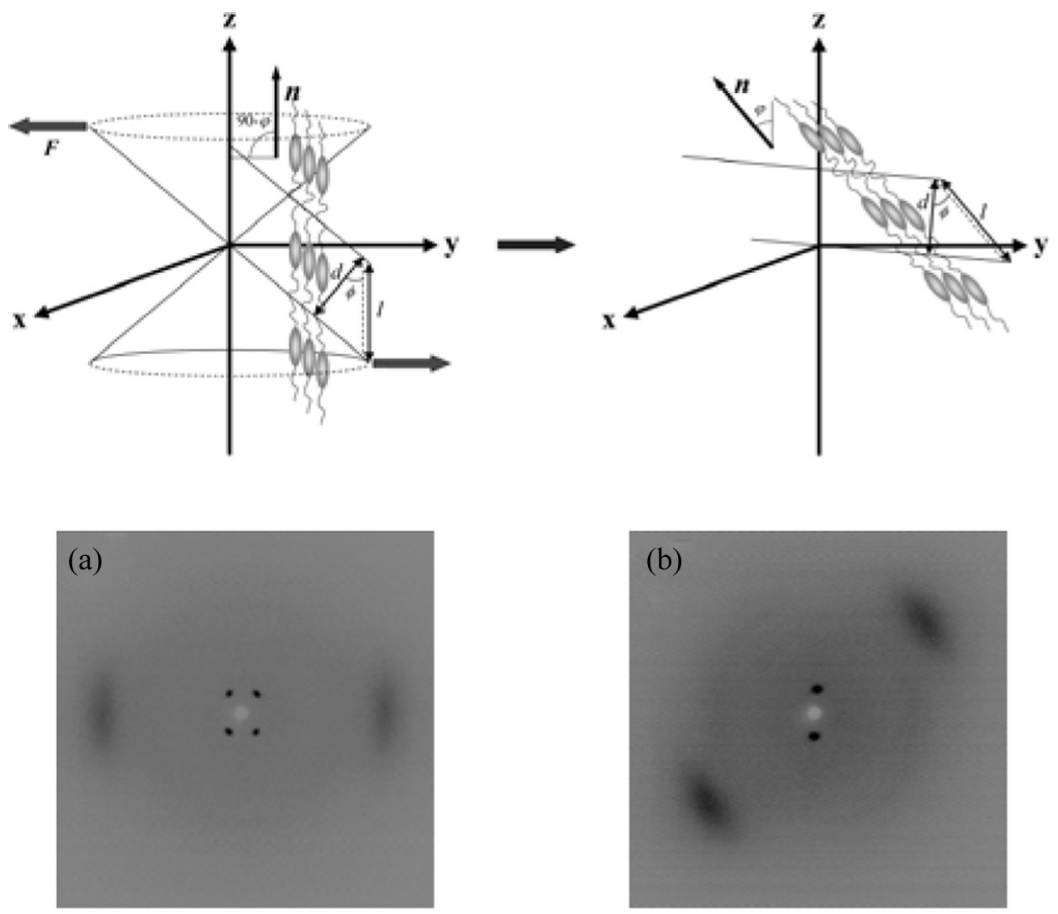

FIGURE 6 Shear deformation process perpendicular to the director on a SmC MCLCE and X-ray pictures at (a) $\gamma=0 \mathrm{rad}$ and (b) $\gamma=0.44 \mathrm{rad}$. 

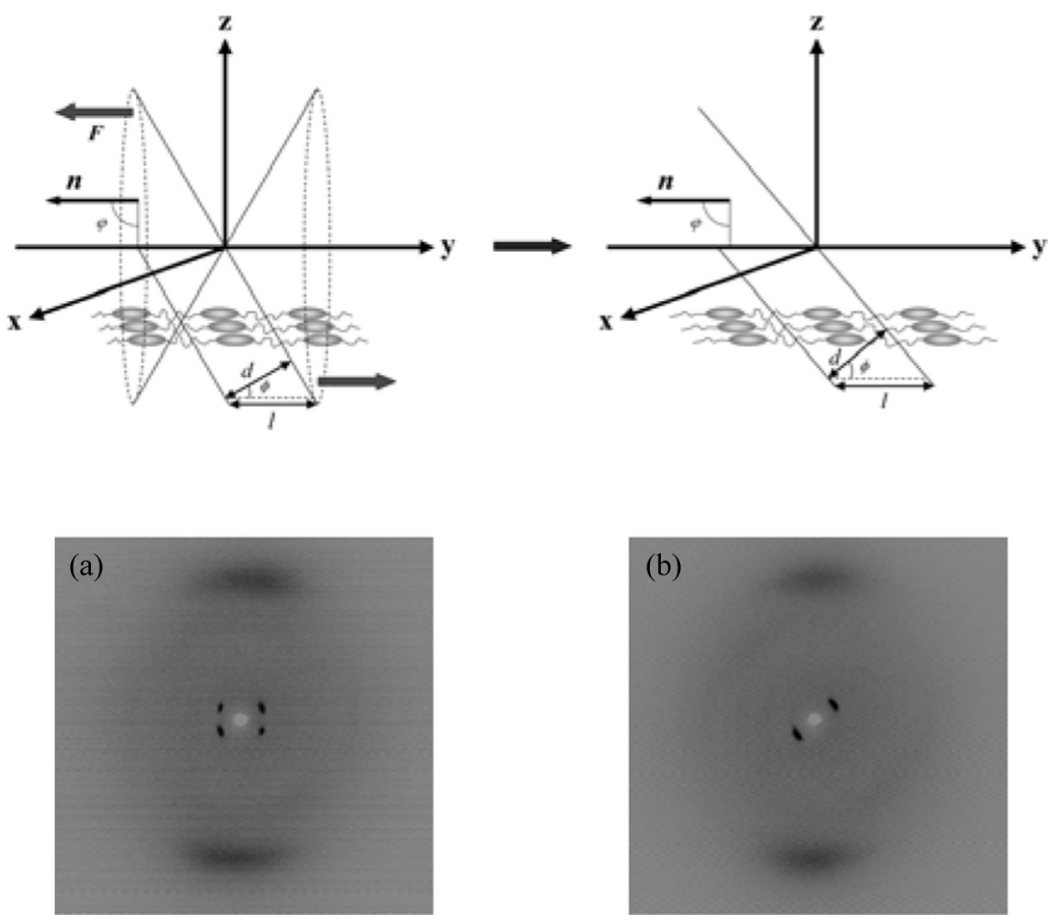

FIGURE 7 Shear deformation process parallel to the director on a $\mathrm{SmC}$ MCLCE and X-ray pictures at (a) $\gamma=0 \mathrm{rad}$ and (b) $\gamma=0.68 \mathrm{rad}$.

become reoriented, where the angle between layer plane and shear angle increases with shear.

If we compare the ratio between the intensities of the WAXS and SAXS with that of the reoriented sample from the previous experiment, the reorientation for the shear process parallel to the director is less efficient. An indication that this orientation process leading to a monodomain is less efficient might also be indicated by the order parameter, which only increases to $S \sim 0.82$ for the shear angle of $\gamma \sim 40^{\circ}$ in contrast to $S \sim 0.87$ for the previous experiment. Above a deformation of $\gamma \sim 25^{\circ}$ the shear process becomes irreversible and the ordered structure is destroyed for $\gamma>40^{\circ}$ by rotation of the director and the layers.

\section{CONCLUSIONS}

The uniaxial deformation of the elastomer parallel to the director causes an increase of the tilt angle without affecting the director orientation, which is accompanied by an increase of the Young modulus 
parallel to the director. Uniaxial deformation perpendicular to the director produces a reorientation of the director parallel to the applied force. This reorientation process also affects the tilt angle of the smectic structure. After complete reorientation a tilt angle is observed that corresponds to the tilt angle of the sample under uniaxial strain parallel to the director.

The shear deformation perpendicular to the director is a reversible process and couples to the layer as well as to the director orientation. A highly ordered monodomain is obtained by this reversible reorientation process. The shear deformation parallel to the director only affects the layer reorientation towards a monodomain while the director orientation remains unchanged. This irreversible process, however, does not produce a highly ordered monodomain.

The basic difference between main- and side-chain elastomers is that in main-chain elastomers the mechanical field always couples to the tilt angle of the $\mathrm{SmC}$ phase structure. This opens new interesting aspects especially for chiral $\mathrm{SmC}^{*}$ elastomers, where the tilt angle determines the optical and ferro-electrical properties.

\section{REFERENCES}

[1] Nishikawa, E. \& Finkelmann, H. (1999). Macromol. Chem. Phys., 200, 312.

[2] Nishikawa, E., Finkelmann, H., \& Brand, H. R. (1997). Macromol. Rapid Commun., $18,65$.

[3] Nishikawa, E. \& Finkelmann, H. (1997). Macromol. Chem. Phys., 198, 1531.

[4] Stannarius, R., Kohler, R., Dietrich, U., Losche, M., Tolksdorf, C., \& Zentel, R. (2002). R. Phys. Rev. E, 65, 041707.

[5] Stannarius, R., Kohler, R., Roessle, M., \& Zentel, R. (2004). Liq. Cryst., 31, 895.

[6] Hiraoka, K., Sagano, W., Nose, T., \& Finkelmann, H. (2005). Macromolecules., 38,7352 .

[7] Hiraoka, K. \& Finkelmann, H. (2001). Macromol. Rapid Commun., 22, 456.

[8] Hiraoka, K., Stein, P., \& Finkelmann, H. (2004). Macromol. Chem. Phys., 205, 48.

[9] Semmler, K. \& Finkelmann, H. (1995). Macromol. Chem. Phys., 196, 3197.

[10] Benne, I., Semmler, K., \& Finkelmann, H. (1994). Macromol. Rapid Commun., 15, 295. 